\title{
Evaluation of a Barrier Repair Cream Containing Pseudo-Ceramide for Practical Use by Hairdressers with Hand Skin Disorders Due to Daily Exposure to Chemical Irritants
}

\author{
Akihiko Takahashi $^{{ }^{*}}$, Annette Kirst ${ }^{2}$, Ulrike Heinrich ${ }^{3}$, Akira Kiyomine ${ }^{2}$, Koichi Ishida ${ }^{1}$, \\ Hagen Tronnier ${ }^{3}$, Heintz Theis $^{2}$, Takahiro Nishizaka ${ }^{1}$, Hisateru Tanabe ${ }^{1}$ \\ ${ }^{1}$ Kao Corporation, Skin Beauty Laboratories, Tokyo, Japan; ${ }^{2}$ Kao Germany, European Research Laboratories, Darmstadt, Germany; \\ ${ }^{3}$ Derma Tronnier, University of Witten-Herdecke, Witten, Germany. \\ Email: "takahashi.akihiko@kao.co.jp
}

Received October $24^{\text {th }}, 2013$; revised November $20^{\text {th }}, 2013$; accepted November $28^{\text {th }}, 2013$

Copyright (C) 2013 Akihiko Takahashi et al. This is an open access article distributed under the Creative Commons Attribution License, which permits unrestricted use, distribution, and reproduction in any medium, provided the original work is properly cited.

\begin{abstract}
Hairdressers are exposed to numerous skin-damaging factors, such as wet work, skin irritants, and thermal changes. Skin protection is a very important factor for the prevention of occupational skin disorders. Therefore, we evaluated a hand cream that was specially formulated for highly stressed skin with regard to its capability to reduce dry, rough, and scaly skin. The objective of this study was to conduct a controlled use test of a barrier repair cream containing pseudo-ceramide and demonstrate its efficacy for hairdressers with dry, sensitive hands and/or occupational skin disorders. A total of 30 hairdressers (29 females and 1 male, aged 20 - 67) participated in the study. All subjects suffered from dry hands. Subjects applied the test cream on their hands for 28 days (four weeks). For each subject, the skin surface topography of the hands was measured by the Surface Evaluation of Living Skin (SELS) system at day 14 (week 2) and day 28 (week 4). Dryness, scaling, cracking, redness, and itchiness were evaluated and graded by experts at week 2 and week 4, and then compared to baselines. All 30 subjects completed the study. A comparison of the changes from baseline to endpoint revealed a statistically significant reduction in skin roughness, scaling, and volume measured according to SELS. These data were confirmed by photographic documentation. The results were further supported by the expert evaluation. For the symptoms dryness and scaling, improvement could be observed in almost all regions of the hands. In addition, according to subjective assessments of overall hand skin condition and the hand cream, subjects reported clear improvement over time (p value below 0.001 ) and good efficacy of the product. In this study, the test hand cream showed excellent skin compatibility. Therefore, the hand cream may be effective for hairdressers with dry, sensitive hands and/or occupational skin disorders.
\end{abstract}

Keywords: Occupational Skin Disorder; Hairdresser; Dermatological Controlled Use Test; Pseudo-Ceramide; Barrier Cream; Topical Hand Care

\section{Introduction}

It is well known that excessive cleansing with detergents is one of the causes of skin dryness and roughness. Consequently, many hairdressers who are exposed to chemical irritants, such as those contained in shampoos and hair dyes, on a daily basis suffer from occupational skin disorders [1,2]. Exogenous occupational irritants that deteriorate the skin barrier are considered one of the

\footnotetext{
*Corresponding author.
}

causes of occupational skin disorders. It is known that stratum cornium intercellular lipids, especially ceramides, play an important role in the maintenance of the skin barrier [3-6], but stratum cornium intercellular lipids are washed away by surfactants [7]. As a result, they lower the skin barrier, leading to skin disorders.

For this reason, barrier cream containing ceramide has been developed in recent years, and its efficacy in occupational skin disorders has been evaluated [8].

Although some researchers have reported on the effi- 
cacy of ceramide barrier cream in dry, scaly skin induced artificially by detergents $[9,10]$, no clinical studies have been conducted on a barrier cream containing ceramide for hairdressers who are exposed to chemicals on a daily basis. Therefore, improvement of hand skin symptoms in hairdressers has not been clinically observed.

We developed a skin barrier cream containing synthesized pseudo-ceramide and allantoin, and carried out a controlled use test in hairdressers who had symptoms of dry, sensitive skin on their hands.

\section{Materials and Methods}

\subsection{Test Product}

A test hand cream formulation, containing pseudo-ceramide (Figure 1) and allantoin, was prepared for this study. INCI declaration: Water (Aqua), Cyclopentasiloxane, Glycerin, Cetyl-PG Hydroxyethyl Palmitamide (Pseudo-ceramide), Hydrogenated Polyisobutene, Neopentyl Glycol Ether, Cetyl Dimethicone, Cholesteryl Isostearate, Eucalyptus Globulus Leaf Extract, Magnesium Sulfate, Allantoin, Methylparaben, Succinic Acid, PEG10 Hydrogenated Castor Oil, Sodium Hydroxide, Tocopheryl Acetate, Bis-Methoxypropylamide Isodocosane. Pseudo-ceramide was synthesized following the procedures in a previous report [11].

\subsection{Selection of Test Subjects}

Thirty healthy hairdressers (29 females and 1 male) aged between 20 and 67 years of age ( $40.5 \pm 12.6$ years) who suffered from dry, sensitive skin on their hands and were able to visit the University of Witten-Herdecke in Germany were selected as test subjects for the study (Table 1).

\subsection{Study Design}

This was a monadic study without any control group. After a three-day regression period (during which subjects were not allowed to use hand care products on their hands), the test subjects applied the test product on their hands, instead of their own hand cream products, at least three times per day over a period of four weeks (28 days). Expert evaluation, instrument measurement, digital photography, and a questionnaire were performed on day 0 (baseline), day 14 (week 2), and day 28 (week 4). The study was realized from April to May 2012.

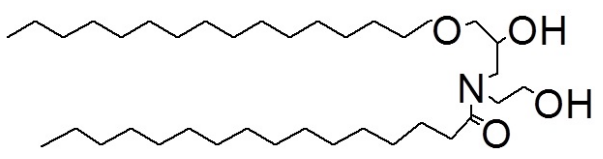

Figure 1. Chemical structure of pseudo-ceramide (Cetyl-PG Hydroxyethyl Palmitamide).

\subsection{Skin Physiological Parameters}

The skin surface topography was measured with the Visioscan ${ }^{\circledR}$ VC98 (Courage \& Khazaka Electronics GmbH, Germany). All subjects were required to undergo a thirtyminute acclimation period $\left(22^{\circ} \mathrm{C}, 40 \%\right.$ relative humidity). The values recorded were means of three measurements.

The Surface Evaluation of Living Skin (SELS) [12] measuring device consists of a measuring head (VisioScan C, Courage \& Khazaka, Cologne) containing two special metal-halogenide lights arranged on opposite sides that uniformly illuminate a $15 \times 17 \mathrm{~mm}$ measuring area of the skin. The spectrum of the lamps as well as their intensity and location had been chosen in such a way that only the skin surface, without reflections of deeper layers, was monitored. Furthermore, unlike with formerly applied procedures with diagonal illumination, with SELS, a distinct representation of the wrinkling is avoided. A CCD-camera, built into the measuring head, takes an image of the skin, which is then transferred as a gray-value bitmap-file by means of the software Skinvisiometer (Courage \& Khazaka, Cologne). The color value for each pixel corresponds to quantification with eight bits, which allows a differentiation into 256 grey values. The value "0" corresponds to the color "black", and the value "255" corresponds to the color "white". By means of the additional software, the SELS skin specific parameters (roughness, scaling, wrinkles, and volume) are then calculated.

\subsection{Expert Evaluation}

To determine skin dryness, scaling, cracking, redness, and itchiness of the hands, a subjective visual evaluation of each test subject was conducted by an expert according to the following four grade scales: $0=$ none, $1=$ slight symptoms, 2 = mild symptoms, and $3=$ moderate symptoms. Dryness, scaling, cracking, redness, and itchiness were recorded for the palm side (palm, fingers, fingertips) and back side (back, fingers, fingertips).

\subsection{Subjective Evaluation}

For the subjective assessment of the test product, cosmetic acceptance and satisfaction of hand skin condition were evaluated. The subjects assessed the cosmetic acceptance and rated the product as "very bad", "bad", "neither good nor bad", "good", or "very good". The subjects' satisfaction with their hand skin condition at baseline, week 2, and week 4 were rated as "not satisfied", "not so satisfied", "somewhat satisfied", "satisfied", and "extremely satisfied".

\subsection{Digital Photography}

To objectively measure the responsiveness to the treat- 

with Hand Skin Disorders Due to Daily Exposure to Chemical Irritants

Table 1. Hand skin condition at baseline visit.

\begin{tabular}{|c|c|c|c|c|c|c|c|}
\hline \multirow{2}{*}{ Symptom } & \multirow{2}{*}{ Grading } & \multicolumn{3}{|c|}{ Front side } & \multicolumn{3}{|c|}{ Back side } \\
\hline & & Hand & Finger & Finger tip & Hand & Finger & Finger tip \\
\hline \multirow{5}{*}{ Dryness } & $0:$ None & 15 & 13 & 14 & 0 & 0 & 3 \\
\hline & 1:Slight & 8 & 7 & 9 & 6 & 7 & 4 \\
\hline & 2:Mild & 4 & 7 & 5 & 14 & 15 & 17 \\
\hline & 3:Moderate & 3 & 3 & 2 & 10 & 8 & 6 \\
\hline & Average & 0.83 & 1 & 0.83 & 2.13 & 2.03 & 1.87 \\
\hline \multirow{5}{*}{ Scaling } & 0:None & 24 & 20 & 24 & 11 & 12 & 16 \\
\hline & 1:Slight & 3 & 8 & 3 & 9 & 12 & 9 \\
\hline & 2:Mild & 1 & 71 & 2 & 7 & 6 & 1 \\
\hline & 3:Moderate & 2 & 1 & 1 & 3 & 0 & 4 \\
\hline & Average & 0.37 & 0.43 & 0.33 & 1.07 & 0.8 & 0.77 \\
\hline \multirow{5}{*}{ Cracking } & 0:None & 30 & 30 & 27 & 26 & 28 & 25 \\
\hline & 1:Slight & 0 & 0 & 2 & 2 & 0 & 2 \\
\hline & 2:Mild & 0 & 0 & 0 & 1 & 2 & 2 \\
\hline & 3:Moderate & 0 & 0 & 1 & 1 & 0 & 1 \\
\hline & Average & 0 & 0 & 0.17 & 0.23 & 0.13 & 0.3 \\
\hline \multirow{5}{*}{ Redness } & 0:None & 30 & 30 & 30 & 12 & 17 & 27 \\
\hline & 1:Slight & 0 & 0 & 0 & 10 & 8 & 2 \\
\hline & 2:Mild & 0 & 0 & 0 & 7 & 5 & 1 \\
\hline & 3:Moderate & 0 & 0 & 0 & 1 & 0 & 0 \\
\hline & Average & 0 & 0 & 0 & 0.9 & 0.6 & 0.13 \\
\hline \multirow{5}{*}{ Itchiness } & 0:None & 26 & 28 & 29 & 13 & 16 & 26 \\
\hline & 1:Slight & 4 & 1 & 0 & 7 & 9 & 2 \\
\hline & 2:Mild & 0 & 1 & 1 & 8 & 5 & 2 \\
\hline & 3:Moderate & 0 & 0 & 0 & 2 & 0 & 0 \\
\hline & Average & 0.13 & 0.1 & 0.07 & 0.97 & 0.63 & 0.2 \\
\hline
\end{tabular}

ment of the highly stressed skin on the hands, photographic documentation was performed (Fotofinder Systems GmbH, Bad Birnbach, Bavaria, Germany).

\subsection{Ethics}

The study protocol was approved by the ethical committee of the University of Witten-Herdecke. All participating test subjects were informed about the objectives and scope of the study and provided written informed consent prior to the study. The study was carried out in accordance with the GCP-Guideline.

\subsection{Statistical Analysis}

A two-sided Wilcoxon signed rank sum test was used for statistical analysis of the SELS and questionnaire data.

The statistical analysis of the visual evaluation by experts was performed by Steel's multiple comparison test to investigate differences in distributions in comparison to baselines.

P-values of less than 0.05 were considered as statisticcally significant.

First, confirm that you have the correct template for your paper size. This template has been tailored for out- 
put on the custom paper size $(21 \mathrm{~cm} * 28.5 \mathrm{~cm})$.

\section{Results}

All thirty enrolled test subjects completed the study. All participants had used their own hand care products on their hands prior to the study, and had dry hand skin at the baseline visit.

The SELS parameters roughness, scaling, wrinkles, and skin volume were evaluated. Skin roughness parameter decreased by $29.7 \%(\mathrm{p}=0.0002)$ after two weeks and by $32.1 \%(\mathrm{P}=0.0003)$ after four weeks of treatment with the test product compared to baseline (Figure 2). Scaling parameter decreased by 51.4\% (p < $0.0001)$ after two weeks and by $49.7 \%(\mathrm{p}<0.0001)$ after four weeks of treatment with the test product compared to baseline (Figure 3). Skin wrinkle parameter decreased

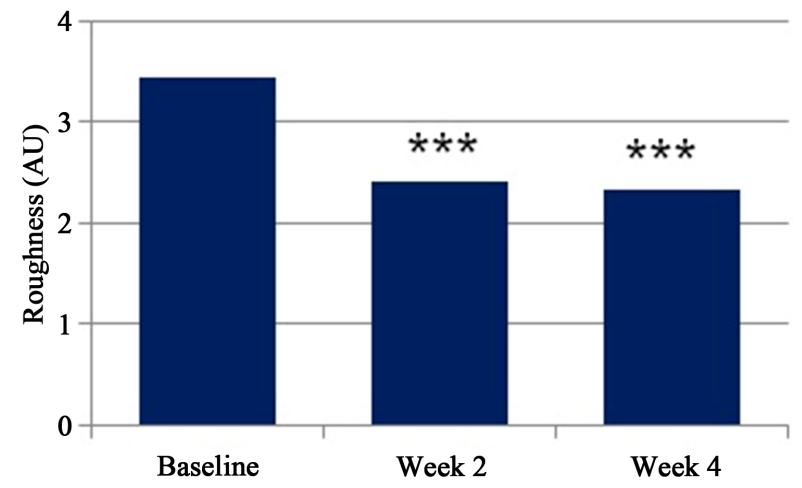

Figure 2. Skin roughness: At baseline and after 2 and 4 weeks of hand cream treatment the skin roughness was assessed. Statistical differences in skin roughness between baseline and after 2 weeks' and 4 weeks' treatment are indicated $\left(^{*} \mathrm{p}<0.05,{ }^{* *} \mathrm{p}<0.01,{ }^{* * *} \mathrm{p}<0.001\right.$ Wilcoxon signedrank test). The bars indicate SD of means for 30 test subjects.

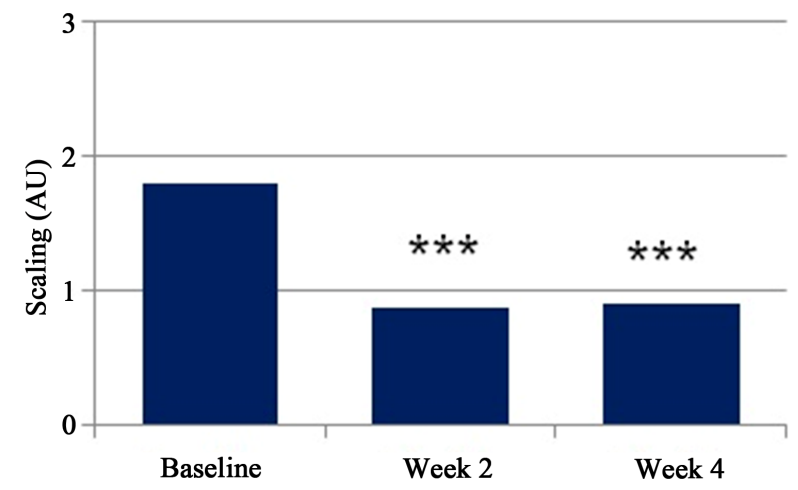

Figure 3. Skin scaling: At baseline and after 2 and 4 weeks of hand cream treatment skin scaling was assessed. Statistical differences in skin scaling between baseline and after 2 weeks' and 4 weeks' treatment are indicated $\left({ }^{*} \mathrm{p}<0.05,{ }^{* *} \mathrm{p}\right.$ $<0.01,{ }^{* * *} p<0.001$ Wilcoxon signed-rank test). The bars indicate SD of means for 30 test subjects. by about 3\% after two weeks and after 4 weeks of treatment with the test product. Thus, there were no statistical significances between skin wrinkles after two weeks ( $\mathrm{p}=$ $0.217)$ and four weeks $(p=0.109)$ compared to baseline (Figure 4). The parameter volume is the virtual amount of liquid which is required to fill the depth. Thus, the smoother the skin, the less virtual liquid is needed to fill the depth. The skin volume significantly decreased by $16.1 \%(\mathrm{p}=0.002)$ after two weeks and by $15.75 \%(\mathrm{p}=$ 0.0239) after four weeks of supplementation (Figure 5).

In addition to the SELS analysis, improvements of the skin were assessed by comparing observations of the dryness, scaling, cracking, redness, and itchiness of the skin by experts at different time intervals.

At baseline, all subjects had skin dryness on the backs of their hands and fingers. Severities of dryness, scaling, cracking, redness, and itchiness on the backs of their hands were worse than those of the palms. The mean

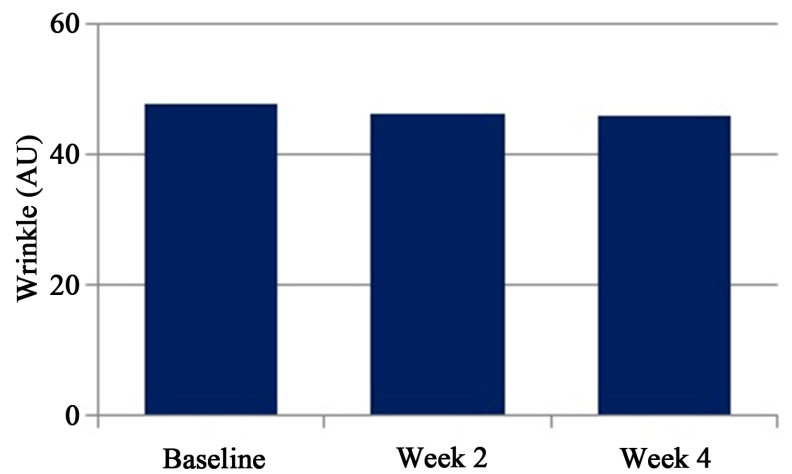

Figure 4. Skin wrinkles: At baseline and after 2 and 4 weeks of hand cream treatment skin wrinkles were assessed. Statistical differences in skin wrinkles between baseline and after 2 weeks' and 4 weeks' treatment are indicated ( ${ }^{*}$ p < $0.05,{ }^{* *} p<0.01,{ }^{* * *} p<0.001$ Wilcoxon signed-rank test). The bars indicate SD of means for 30 test subjects.

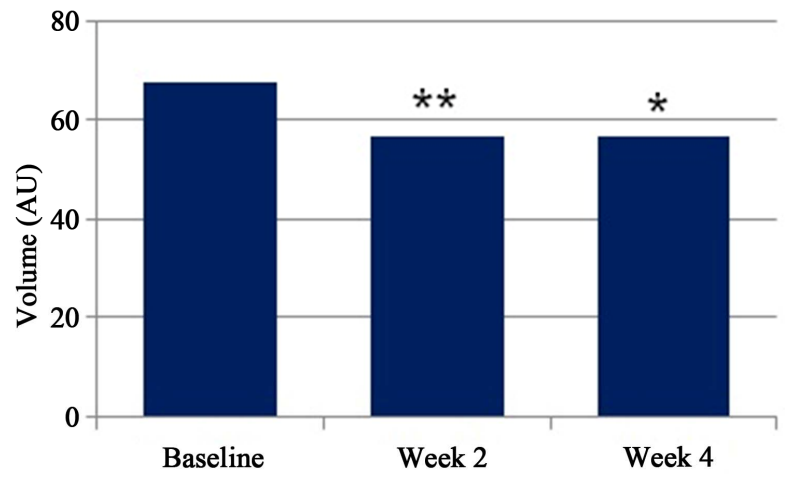

Figure 5. Skin volume: At baseline and after 2 and 4 weeks of hand cream treatment skin volume was assessed. Statistical differences in skin volume between baseline and after 2 weeks' and 4 weeks' treatment are indicated ( ${ }^{*}$ p < $0.05,{ }^{* *} p<0.01,{ }^{* * *} p<0.001$ Wilcoxon signed-rank test). The bars indicate SD of means for 30 test subjects. 

with Hand Skin Disorders Due to Daily Exposure to Chemical Irritants

grade of dryness on the backs of hands went from 2.13 at baseline down to 1.00 at week 2, and then further down to 0.20 at week 4 . Along with the improvement of hand skin dryness, the mean grades of scaling, redness, and itchiness also decreased (Figure 6).

No side effects (adverse reactions) were observed

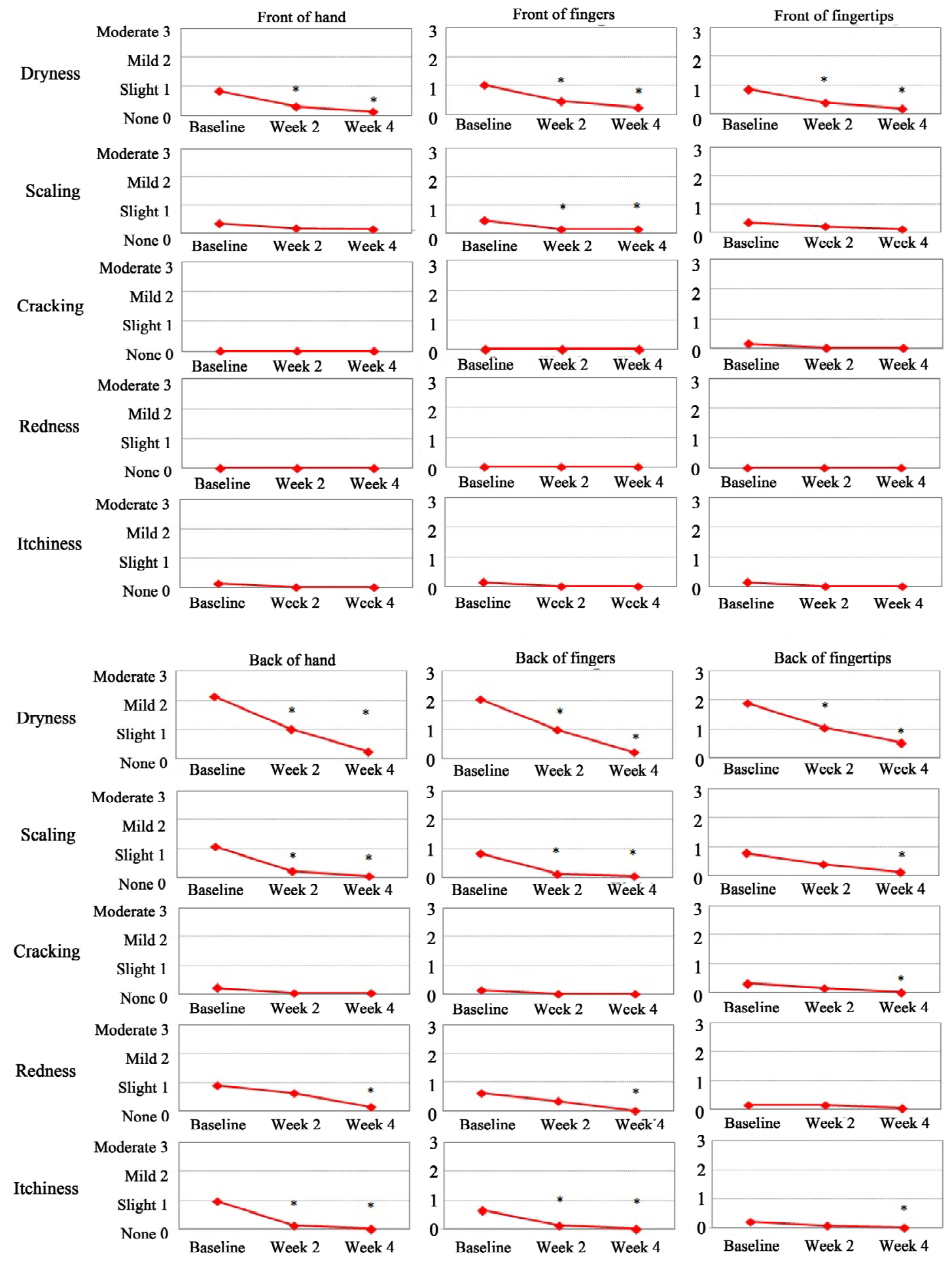

Figure 6. Expert gradings: At baseline and after 2 and 4 weeks of hand cream treatment, experts graded 6 regions of the hands. Statistical differences in expert gradings between baseline and after 2 weeks' and 4 weeks' treatment are indicated ( ${ }^{*} p$ $<0.05$ Steel's multiple comparison test). 
throughout the study period for all test subjects. In regards to skin compatibility of the test product, $70.0 \%$ [21] of the test subjects were very satisfied and 30.0\% [9] were satisfied. Therefore, all test subjects were pleased with skin compatibility, indicating that the test product was very good in terms of skin compatibility (Table 2).

According to subjective assessments of overall hand skin condition, subjects reported improvement throughout the test period (Figure 7). In terms of cosmetic acceptance, $93.3 \%$ of test subjects rated the test product as "very good" or "good" at week 4 (Table 3).

The positive effects on the skin of the hands shown by skin physiological parameters measured by the SELS method and by expert evaluation, as well as the noted subjective assessments, were confirmed by digital photographic documentation. A selection of representative digital photographs is shown in Figure 8. Before the

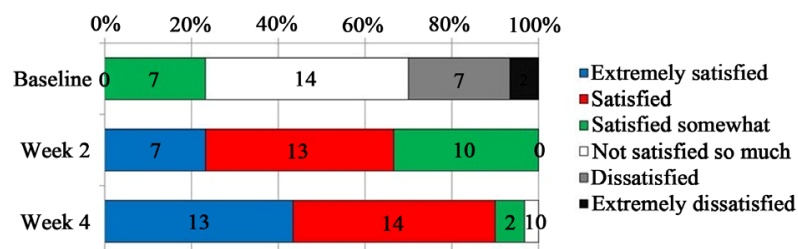

Figure 7. Changes in subjective assessment of satisfaction of hand skin condition: After daily applications of test hand cream, subjects' satisfaction of their hand skin condition improved significantly (Week 2 versus Baseline: $\mathbf{p}<\mathbf{0 . 0 0 1}$, Week 4 versus Baseline: $p<0.001$ Wilcoxon signed-rank test).

Table 2. Skin compatibility.

\begin{tabular}{ccc}
\hline & Number & $\%$ \\
\hline Very good & 21 & 70 \\
Good & 9 & 30 \\
Fair & 0 & 0 \\
Satisfactory & 0 & 0 \\
Unsatisfactory & 0 & 0 \\
Insufficient & 0 & 0 \\
\hline
\end{tabular}

Table 3. Subjects' assessment of the clinical acceptance of the test hand cream.

\begin{tabular}{cccc}
\hline & $\mathrm{N}$ & $\%$ & Cumulative \% \\
\hline Very good & 13 & 43.3 & 43.3 \\
Good & 15 & 50 & 93.3 \\
Neither goog nor bad & 2 & 6.7 & 100 \\
Bad & 0 & 0 & 100 \\
Very bad & 0 & 0 & 100 \\
\hline
\end{tabular}
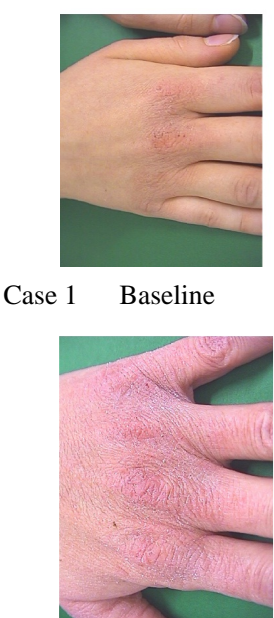

Case 2 Baseline
Case 1 Baseline

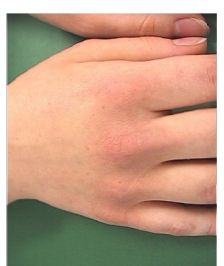

Week 2

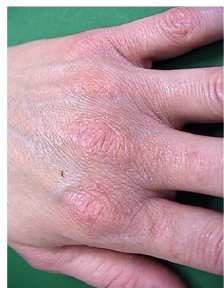

Week 2

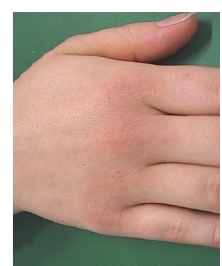

Week 4

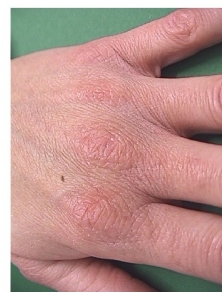

Week 4
Figure 8. Photographic documentation: At baseline visit, the skin was rough, flaky, and dry. However, after daily applications of the test hand cream, the skin on the hands appeared shiny, smooth, and well maintained.

treatment, the skin was rough and dry. However, after daily application of the test product for four weeks, the skin on the hands appeared shiny, smooth, and well maintained.

\section{Discussion}

Hairdressers are exposed to numerous skin-damaging factors, such as wet work, chemical irritants, and thermal changes. In fact, $87 \%$ of the subjects of this study did not wear gloves while shampooing hair, and $77 \%$ of them did not wear gloves while perming hair (Table 4). Thus, skin protection by hand care treatment is a very important factor for the prevention of occupational skin disorders.

Ceramide is well known as one of the stratum cornium intercellular lipids that play an important role in the maintenance of skin barrier function [3]. With a decrease in the amount of ceramide and other stratum cornium intercellular lipids, the skin barrier function lowers on atopic dermatitis [4]. Decreased ceramide level is caused by an abnormality in ceramide metabolism in atopic dermatitis $[13,14]$. It has been reported that application of barrier repair cream containing ceramide improves atopic symptoms along with improvement of the skin barrier function as measured by transepidermal water loss $[15,16]$.

However, detergents are known to cause dry skin problems since they facilitate the flowing out of stratum cornium intercellular lipids [7]. Previous reports have shown the efficacy of treatment with topical creams containing ceramide in cases of dry skin induced by detergents [8,9], but there have been no reports on the 
with Hand Skin Disorders Due to Daily Exposure to Chemical Irritants

Table 4. Glove use ratio of daily duties.

\begin{tabular}{cccccc}
\hline & \multicolumn{5}{c}{$\mathrm{N}(\%)$} \\
\hline Duty & Hair shampoo & Hair coloring & Bleaching & Hair perm (acid) & Hair perm (alkali) \\
\hline with globe & $4(13.3)$ & $27(90.0)$ & $22(73.3)$ & $7(23.3)$ & $7(23.3)$ \\
without globe & $26(86.7)$ & $3(10.0)$ & $8(26.7)$ & $23(76.7)$ & $23(767.7)$ \\
\hline
\end{tabular}

clinical efficacy of ceramide cream in occupational skin disorders of hairdressers who are exposed to chemical irritants on a daily basis. Therefore, a hand cream that contains pseudo-ceramide and is formulated for highly stressed skin of the hands is evaluated with regard to its capability of reducing dry, rough, and scaly skin.

A comparison of changes from baseline to endpoint revealed a statistically significant reduction in skin roughness, scaling, and volume measured according to SELS. These data were confirmed by photographic documentation.

The results were further supported by expert evaluation. For the symptoms dryness and scaling, improvements could be observed in almost all regions of the hands.

In addition, self-assessment showed that overall hand skin condition clearly improved over time ( $\mathrm{p}$ value below 0.001), and test subjects reported good efficacy of the test product.

Since problem skin requires special skin care, skin care products have to be mild with excellent skin compatibility, preventing skin irritations and dryness. In this study, the test hand cream showed excellent skin compatibility.

Since this study was a single-test without any controls, we could not definitively declare the efficacy of the test hand cream. However, though all of the subjects continued their daily work tasks, including shampooing and perming hair, throughout the test period and had used commercial hand care products until the study started, the clinical scores and SELS measurements improved significantly. In addition, by self-assessment, subjects reported improved hand skin condition throughout the test period. These results suggest that the test hand cream is effective for dry hand skin problems in hairdressers.

In order to confirm the efficacy for occupational hand skin eczema, we should conduct a double-blind study using placebo as a control and measure skin barrier function to clearly demonstrate that the effects of the test product are due to pseudo-ceramide and its ability to maintain skin barrier function.

\section{Acknowledgements}

The authors would like to thank Mrs. Dipl. Stat. C. Theek for her assistance, including statistical consulting and evaluation of the data, and all the hairdresser subjects, whose cooperation made this investigation possible.

\section{REFERENCES}

[1] A. Worth, S. H. Arshad and A. Sheikh, "Occupational Dermatitis in a Hairdresser,” British Medical Journal, Vol. 335, 2007, p. 399.

http://dx.doi.org/10.1136/bmj.39252.524317.94

[2] M. L. Lind, M. Albin, J. Brisman, et al., "Incidence of Hand Eczema in Female Swedish Hairdressers," Occupational and Environmental Medicine, Vol. 64, No. 3, 2007, pp. 191-195. http://dx.doi.org/10.1136/oem.2005.026211

[3] P. M. Elias, "Epidermal Lipids, Barrier Function, and Desquamation,” The Journal of Investigative Dermatology, Vol. 80, 1983, pp. 44s-49s.

http://dx.doi.org/10.1038/jid.1983.12

[4] G. Imokawa, A. Abe, K. Jin, et al., "Decreased Level of Ceramides in Stratum Cornium of Atopic Dermatitis: An Etiologic Factor in Atopic Dry Skin?” The Journal of Investigative Dermatology, Vol. 96, 1991, pp. 523-526. http://dx.doi.org/10.1111/1523-1747.ep12470233

[5] K. C. Madison, "Barrier Function of the Skin: 'La Raison d'Être' of the Epidermis," The Journal of Investigative Dermatology, Vol. 121, 2003, pp. 231-241. http://dx.doi.org/10.1046/j.1523-1747.2003.12359.x

[6] T. Dirschka, C. Szliska, J. Jackowski and H. Tronnier, "Impaired Skin Barrier and Atopic Diathesis in Perioral Dermatitis,” Journal der Deutschen Dermatologischen Gesellschaft, Vol. 1, No. 3, 2003, pp. 199-203. http://dx.doi.org/10.1046/j.1610-0387.2003.02039.x

[7] G. Imokawa, "Surfactant-Induced Depletion of Ceramides and Other Intercellular Lipids: Implication for the Mechanism Leading to Dehydration of the Stratum Corneum,” Exogenous Dermatology, Vol. 3, 2004, pp. 81-98. http://dx.doi.org/10.1159/000086158

[8] L. Halkier-Sorensen and K. Thestrunp-Pedersen, “The Efficacy of Moisturizer (Locobase) among Cleaners and Kitchen Assistants during Everyday Exposure to Water and Detergents," Contact Dermatitis, Vol. 29, No. 5, 1993, pp. 266-271. http://dx.doi.org/10.1111/j.1600-0536.1993.tb03563.x

[9] M. Kucharekova, J. Schalkwijk, P. C. M. Kerkhof, et al., "Effect of a Lipid-Rich Emollient Containing Ceramide3 in Experimentally Induced Skin Barrier Dysfunction,” Contact Dermatitis, Vol. 46, No. 6, 2002, pp. 331-338. http://dx.doi.org/10.1034/j.1600-0536.2002.460603.x

[10] M. Loden and E. Barany, "Skin-Identical Lipids versus 
Petrolatum in the Treatment of Tape-Stripped and Detergent-Perturbed Human Skin,” Acta Dermato-Venereologica, Vol. 80, 2000, pp. 412-415. http://dx.doi.org/10.1080/000155500300012774

[11] G. Imokawa, S. Akasaki, O. Kuno, et al., "Function of Lipids on Human Skin,” Journal of Dispersion Science and Technology, Vol. 10, No. 4-5, 1989, pp. 617-641. http://dx.doi.org/10.1080/01932698908943190

[12] H. Tronnier, M. Wiebusch, U. Heinrich, et al., "Surface Evaluation of Living Skin,” Advances in Experimental Medicine and Biology, Vol. 455, 1999, pp. 507-516. http://dx.doi.org/10.1007/978-1-4615-4857-7_75

[13] Y. Murata, J. Ogata, Y. Higaki, et al., "Abnormal Expression of Sphingomyelin Acylase in Atopic Dermatitis: An Etiologic Factor for Ceramide Deficiency?” The Journal of Investigative Dermatology, Vol. 106, 1996, pp. 1242-1249.

http://dx.doi.org/10.1111/1523-1747.ep12348937
[14] J. Hara, K. Higuchi, R. Okamoto, et al., "High-Expression of Sphingomyelin Deacylase Is an Important Determinant of Ceramide Deficiency Leading to Barrier Disruption in Atopic Dermatitis,” The Journal of Investigative Dermatology, Vol. 115, 2000, pp. 406-413. http://dx.doi.org/10.1046/j.1523-1747.2000.00072.x

[15] S. L. Chamlin, J. Kao, I. J. Frieden, et al., "CeramideDominant Barrier Repair Lipids Alleviate Childhood Atopic Dermatitis: Changes in Barrier Function Provide a Sensitive Indicator of Disease Activity," Journal of the American Academy of Dermatology, Vol. 47, No. 2, 2002, pp. 198-208. http://dx.doi.org/10.1067/mjd.2002.124617

[16] M. Mao-Qiang, K. R. Feingold and P. M. Elias, "Influence of Exogenous Lipids on Permeability Barrier Recovery in Acetone-Treated Murine Skin,” JAMA Dermatology, Vol. 129, No. 6, 1993, pp. 728-738. http://dx.doi.org/10.1001/archderm.1993.0168027006600 $\underline{8}$ 